\title{
Original optical metrologies of large components
}

\author{
B. Cimma, D. Forest, P. Ganau, B. Lagrange, J.M. Mackowski, C. Michel, J.L. Montorio, \\ N. Morgado, R. Pignard, L. Pinard* , A. Remillieux \\ SMA - VIRGO 22, Boulevard Niels Bohr 69622 - Villeurbanne Cedex France
}

\begin{abstract}
The coating deposition on large optical components (diameter $350 \mathrm{~mm}$ ) has required the development of new metrology tools at $1064 \mathrm{~nm}$. To give realistic values of the optical performances, the whole surface of the component needs to be scanned. Our scatterometer (commercial system) has been upgraded to support large and heavy samples. The other metrology tools are prototypes we have developed. We can mention the absorption (photothermal effect) and birefringence bench, a control interferometer equipped with an original stitching option, the optical profilometer (RMS roughness and small defect measurements). A detailed description of these metrology benches will be exposed. Their sensitivity, accuracy and capability to map the optical properties of substrates or mirrors will be discussed. We will describe the recent developments: the stitching option adapted to the Micromap profilometer to measure the RMS roughness on larger area (exploration of a new spatial frequency domain), the accurate bulk absorption calibration.
\end{abstract}

Keywords: Optical metrology, scattering, absorption, birefringence, roughness, stitching interferometry

\section{INTRODUCTION}

Some experiment like laser interferometer for gravitational waves detection (VIRGO for example) needs large and heavy optics having extremely low optical loss. To be able to coat such large components, we have constructed a large Ion Beam Sputtering (IBS) deposition system $\left(2.4 * 2.4 * 2.2 \mathrm{~m}^{3}\right)$. At the present time, the IBS process is the only one, which can produce low loss coatings (absorption and scattering $<5 \mathrm{ppm}$ (part per million)).

The optimization of the IBS process was possible only because, close to the coater, we have developed sensitive optical metrology tools. The coating metrology is fully part of the global process. In the past, it allowed us to improve rapidly the coating performances and to reach a very high quality level ${ }^{1,2}$.

To avoid contamination of the mirrors, which can affect the measurements, all the metrology tools are placed in a class 1 clean room.

The originality of these optical benches consists in the fact that mappings are possible on area up to $\varnothing 400 \mathrm{~mm}$. Thus, the entire surface of substrates or multidielectric mirrors can be characterized: thus, very realistic measurements are made. Another requirement was to have metrology tools capable to measure very low optical loss.

In the following paragraphs, we will describe in detail the metrology systems we have. Their sensitivity, accuracy and capability to map optical properties of substrates or mirrors will be discussed. The optical systems concerned are a scatterometer, an absorption and birefringence bench, a control interferometer with its stitching option and an optical profilometer.

\section{SCATTERING MEASUREMENT}

The scatterometer is a commercial CASI type instrument (Complete Angle Scan Instrument) able to measure scattering levels lower than $1 \mathrm{ppm}$ (BRDF noise floor of $10^{-9} \mathrm{sr}^{-1}$ ). The BRDF (Bi-directional Reflectance Distribution function) can be measured at one point of the sample (beam size $1 \mathrm{~mm}$ ) as a function of the scattered angle $\theta_{\mathrm{s}}$. The detector sweeps around the sample in the incident plane measuring scattered and specular light. The instrument background is measured separately and compared to the sample data.

This system is perfectly linear on 12 decades (maximal intensity variation between the specular beam and the scattered signal). 
"pinard@ipnl.in2p3.fr; phone: 33-4-72-43-26-68; fax: 33-4-72-43-26-79; http://lyoinfo.in2p3.fr/sma/Sma.htm

To have realistic measurements, a BRDF mapping of the entire sample surface is measured to take into account every possible coating or substrate defect. This has implied an upgrade of the piloted scatterometer sample holder to realize mappings up to $\varnothing 400 \mathrm{~mm}$ and to support heavy samples $(40 \mathrm{~kg})$.

Two typical examples of scattering maps are shown on the Figure 1. The first sample (machined metallic block) has a very high average scattering level $\left(1.210^{-2} \mathrm{sr}^{-1}\right.$ on $\varnothing 250 \mathrm{~mm}$ or \# 1\%) and the second one (high reflective multilayer IBS coatings deposited on a micropolished substrate) shows a very low scattering level (4 ppm on $150^{*} 150 \mathrm{~mm}^{2}$ ).
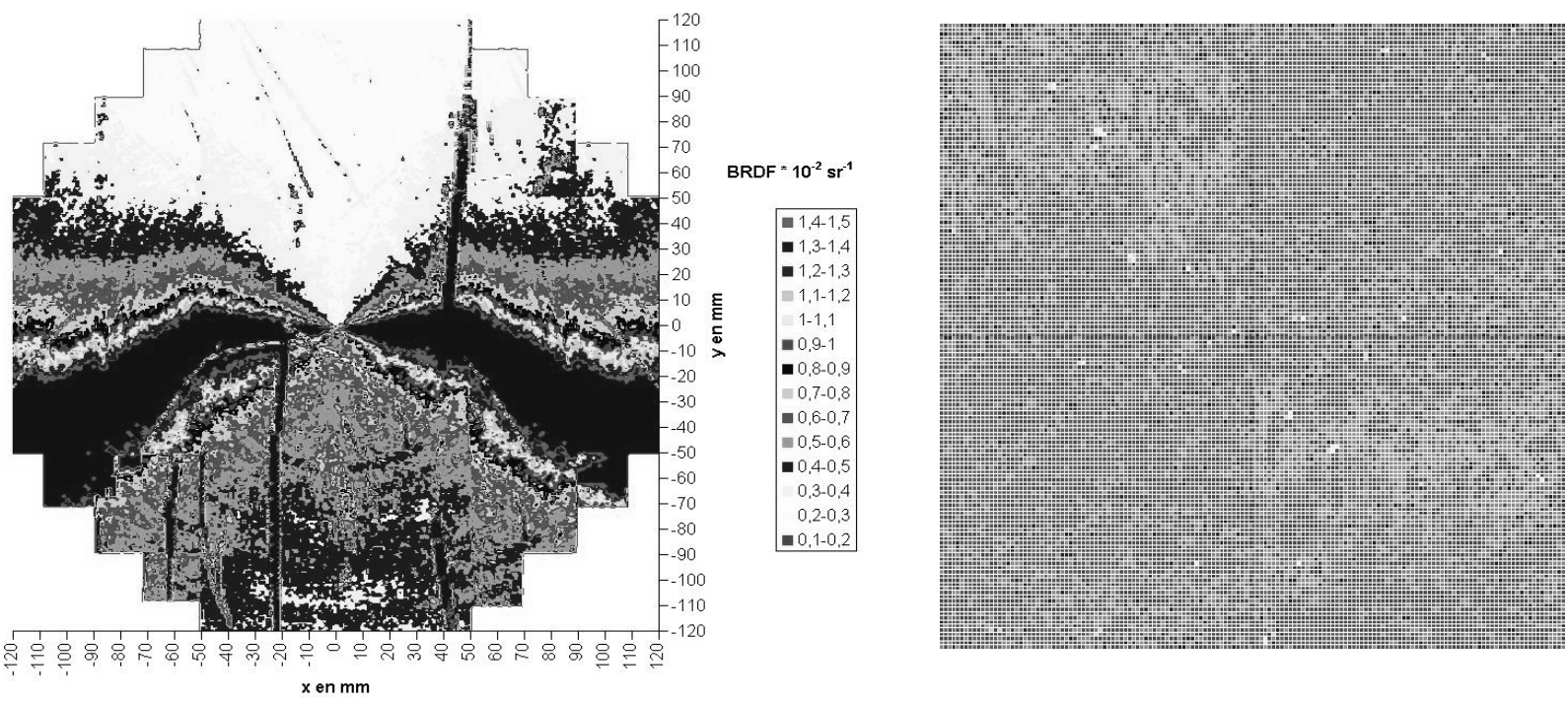

Figure 1 : Scattering maps of a metallic block (left) and of a IBS large mirror (right)

This system can also be used as a very sensitive wattmeter to determine very low transmission values $(\mathrm{T}<1 \mathrm{ppm})$. Transmission maps give a lot of information as, for example, the coating uniformity on large diameter.

Finally, as the laser beam of the system is S polarized, we can separate the scattering coming from both substrate surfaces (roughness, small defects) and the one coming from the bulk material (inclusions, bubbles).

According to the Rayleigh-Rice theory, the BRDF is defined for a polished, cleaned and reflective sample by the formula $^{3,4}$ :

$$
\mathrm{BRDF}=\frac{16 \pi^{2}}{\lambda^{4}} \cos \theta_{\mathrm{i}} \cos ^{2} \theta_{\mathrm{s}} \mathrm{Q} \mathrm{S}\left(\mathrm{f}_{\mathrm{x}}, f_{\mathrm{y}}\right)
$$

- $S\left(f_{x}, f_{y}\right)$ is the power spectral density

- $\theta_{\mathrm{i}}$ is the incident angle

- $\theta \mathrm{s}$ is the scattered angle

- $\mathrm{Q}$ is the polarization factor.

The Q factor is a function of the dielectric constant of the sample $\varepsilon$, of the incident angle $\theta_{\mathrm{i}}$, of the scattered angles $(\theta \mathrm{s}$, $\varphi s)$. Moreover, it depends on the incident polarization (S) and on the polarization analyzed at the detector (S or P).

The BRDF measurement is done in the incident plane defined by the incident beam and the normal to the sample. That means that $\varphi \mathrm{s}=0$. The $\mathrm{Q}$ factor has two components $\mathrm{Q}_{\mathrm{SS}}$ and $\mathrm{Q}_{\mathrm{SP}}$. $\mathrm{Q}_{\mathrm{SP}}$ is defined by the formula:

$$
\mathrm{Q}_{\mathrm{SP}}=\left|\frac{(\varepsilon-1) \sqrt{\left(\varepsilon-\sin ^{2} \theta_{\mathrm{s}}\right) \sin \varphi_{\mathrm{S}}}}{\left(\cos \theta_{i}+\sqrt{\left.\left(\varepsilon-\sin ^{2} \theta_{\mathrm{i}}\right)\right)\left(\varepsilon \cos \theta_{\mathrm{s}}+\sqrt{\left.\left(\varepsilon-\sin ^{2} \theta_{\mathrm{i}}\right)\right)}\right.}\right.}\right|^{2}
$$


In the incident plane, the $\mathrm{Q}_{\mathrm{sp}}$ factor is equal to zero, but not the $\mathrm{Q}_{\mathrm{ss}}$ factor which is defined by the formula:

$$
\mathrm{Q}_{\mathrm{SS}}=\left|\frac{(\varepsilon-1) \cos \varphi_{\mathrm{S}}}{\left(\cos \theta_{i}+\sqrt{\left.\left(\varepsilon-\sin ^{2} \theta_{\mathrm{i}}\right)\right)\left(\cos \theta_{\mathrm{s}}+\sqrt{\left.\left(\varepsilon-\sin ^{2} \theta_{\mathrm{i}}\right)\right)}\right.}\right.}\right|^{2}
$$

This calculation proves that, in the incident plane, a smooth surface does not scatter light, which is $\mathrm{P}$ polarized. This statement is true only if the detector has a small aperture in front of it (this is the case of the CASI scatterometer).

Thus, in the case of a well cleaned sample micropolished on both side, the P polarized scattered light only comes from the bulk material of the sample (bubbles).

\section{ABSORPTION MEASUREMENT}

\subsection{Experimental setup}

The absorption measurement bench (Fig. 2) is based on the photothermal deflection technique ${ }^{5}$.

\subsubsection{The pump beam}

The pump beam is a Continuous Wave $30 \mathrm{~W}$ YAG: $\mathrm{Nd}^{3+}$ laser at $1064 \mathrm{~nm}$ (SL905T model from Spectron). It is equipped with the LS101 light stabilizer that reduces power fluctuations. The Laser stability is $1.5 \%$ RMS. The pump beam is modulated thanks to a mechanical chopper at $210 \mathrm{~Hz}$. This frequency gives the best signal to noise ratio in our specific environment. The beam then propagates through a beam steerer which lifts it at $84 \mathrm{~cm}$ above the optical table. This height is necessary for the measurement of large optics. The beam steerer is composed of two high reflectivity mirrors made at SMA-VIRGO that together do not diminish the pump power more than $0.1 \%$. The pump laser is then focused on the sample. The sample surface is perpendicular to the beam. The pump waist size is $35 \mu \mathrm{m}$.

\subsubsection{The probe beam}

The probe is a low power Helium-Neon laser which is focused on the same point of the sample as the pump. The angle between probe and pump is $3.5^{\circ}$ and the interaction length is $3.4 \mathrm{~mm}$. This value is rather high in order to have a high bulk absorption sensitivity. The probe laser and the focal lenses of both probe and pump are all together mounted on a translation stage ( $\mathrm{Z}$ axis) perpendicular to the surface of the sample. This configuration allows to scan the bulk absorption versus the depth of the sample on a $150 \mathrm{~mm}$ course. The axis of the translation stage is exactly parallel to the direction of the pump beam so that scanning the sample depth does not affect the alignment of the bench. The deviation of the probe is measured with the help of a lock-in amplifier and a quadrants detector placed at $360 \mathrm{~mm}$ behind the interaction region of probe and pump. This long distance is favorable to a high deviation signal.

\subsubsection{Filtering and trapping of the pump beam}

Due to the small angle between probe and pump, the pump beam is not very well separated from the quadrants detector $(25 \mathrm{~mm})$ and might illuminate and heat it. This spurious signal might have severe effect on the sensitivity of the absorption measurement, because it is modulated at the same frequency as the probe deviation and can't be eliminated by the lock-in amplifier. Therefore, we have placed two high reflectivity (HR) mirrors at $1064 \mathrm{~nm}$ in front of the quadrants detector. The low loss HR mirrors are manufactured at SMA-VIRGO. They transmit the probe beam at 633 $\mathrm{nm}$ but not the pump beam at $1064 \mathrm{~nm}$ (less then $10^{-10}$ ). Moreover, less than $1 \mathrm{ppm}$ of the pump beam is absorbed, preventing any heating of the detector. In addition to these filters, it is necessary to deviate the pump beam before it strikes the mount of the detector, otherwise it would be heated as well as the air in front of the detector, hence the deviation of the probe would be modified. We produced a $80 \mathrm{~mm}$ diameter HR mirror at $1064 \mathrm{~nm}$ in order to deviate efficiently all the $1064 \mathrm{~nm}$ light around the detector towards a light trap located at more than one meter from the detector. The pump light reflected by the surface of the sample is also deviated and trapped the same way, as well as the light reflected by the mechanical chopper. Elimination of the spurious pump laser is a key point as regards the sensitivity of the absorption measurement. By trapping and filtering it, we succeeded in improving the sensitivity of the bench by more than two orders of magnitude. 


\subsubsection{Positioning stages}

The original feature of this bench is its ability to scan the absorption on samples as large as $400 \mathrm{~mm}$ in diameter with a high accuracy. We have measured that to have absorption errors less than $1 \%$ during a scanning, due to the defects of the positioning stages, it is necessary that the surface of the sample always stays precisely in the same vertical plan with a precision of $+/-100 \mu \mathrm{m}$. Otherwise, if the measured point moves in the direction of the pump beam during a mapping, it will not stay precisely in the interaction region where pump and probe beams are focalized. This is mostly important for surface absorption where the coating thickness is in the $\mu \mathrm{m}$ range. In order to achieve this severe specification a 400 $\mathrm{kg}$ marble is used to ensure the rigidity of the positioning system. Moreover, the rolling of all the stages is less than 90 $\mu \mathrm{rad}$. For the horizontal translation stage (X axis), a linear motor has been chosen because it is particularly suited for cleanroom use as it produces no particulates. Its travel length is $1500 \mathrm{~mm}$. It is used for the absorption mappings as well as for the birefringence and the large wavefront measurements and the loading of the samples. Its positioning repeatability is less than $2 \mu \mathrm{m}$. Two vertical translation stages are used for the $\mathrm{Y}$ axis motion. Their travel length is 500 $\mathrm{mm}$ and their positioning repeatability is less than $2 \mu \mathrm{m}$ too. The sample holder is a gimbal mount with polar and azimuthal manual adjustment. The resolution of the adjustment is $0.15 \mu \mathrm{rad}$. It is very important to have the $\mathrm{X}$ and $\mathrm{Y}$ translation axis exactly parallel to the surface of the sample during a mapping run. This operation is achieved with a Fizeau interferometer placed in front of the sample.

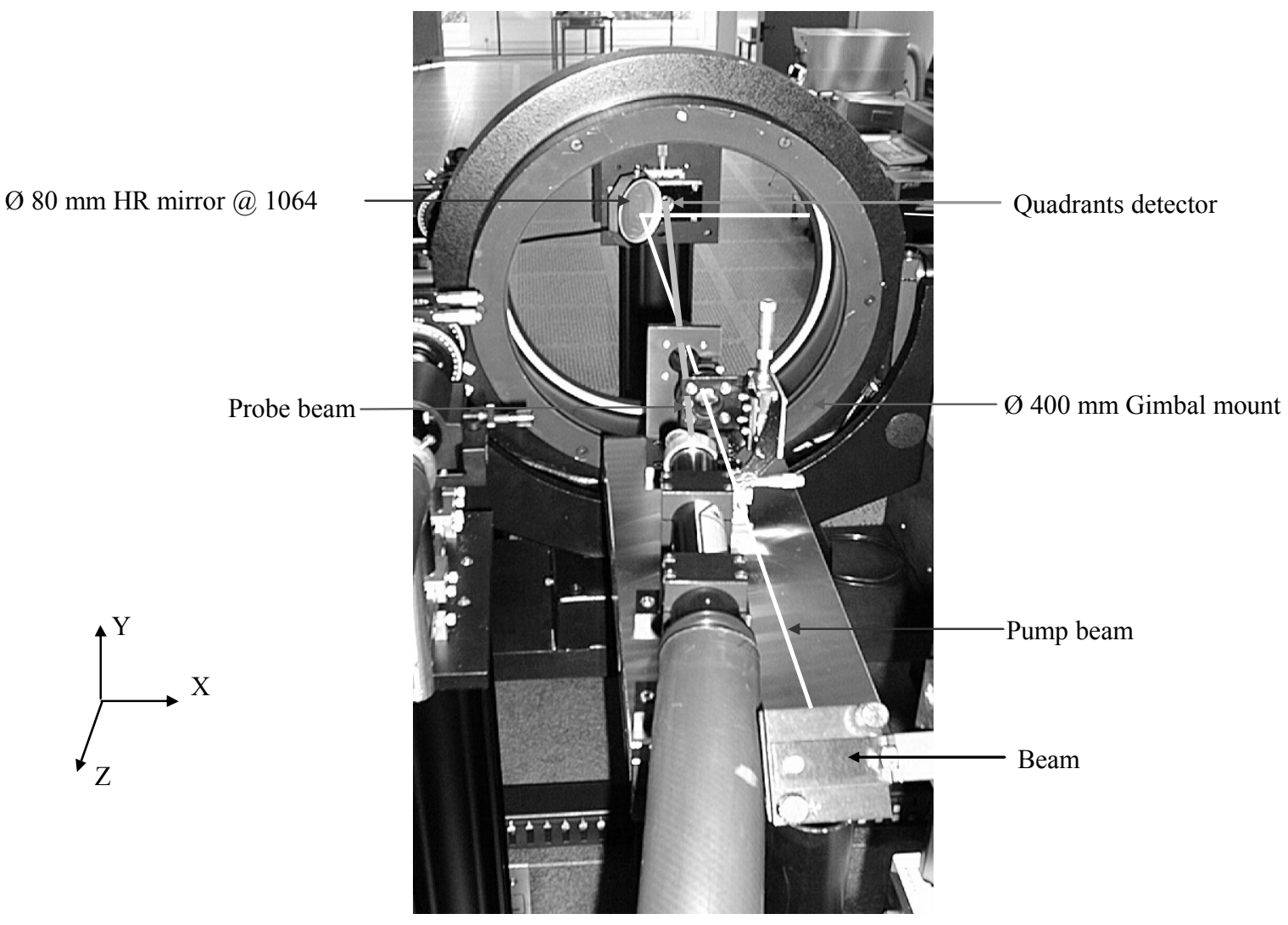

Figure 2: Absorption bench for large components

\subsection{Coating absorption}

\subsubsection{Standard}

The photothermal deflection technique is a very powerful technique for relative absorption measurement. It also allows absolute absorption measurement, if the absorption of a standard is precisely known. The coating absorption $A$ can be defined as follows ${ }^{6}$ : 


$$
A=\left[1-\exp \left(-A_{0}\right)\right] \cdot \frac{\delta V}{\delta V_{0}} \cdot \frac{V_{0}}{V}
$$

where,

- $A_{0}$ is the coating absorption of a well known standard.

- $\delta V$ and $\delta V_{0}$ are the AC voltages relative to the probe deviation of sample and standard respectively.

- $V$ and $V_{0}$ are the DC voltages of the quadrant detector of sample and standard respectively.

The uncertainty on the value of the coating absorption measurement is therefore:

$$
\frac{d A}{A}=\frac{\exp \left(-A_{0}\right)}{\left[1-\exp \left(-A_{0}\right)\right]} \cdot d A_{0}+\frac{d(\delta V)}{\delta V}+\frac{d(\delta V)_{0}}{\delta V_{0}}+\frac{\delta V_{0}}{V_{0}}+\frac{\delta V}{V}
$$

A great effort has been made for the characterization of a standard for the coating absorption. A specific monolayer of $\mathrm{TaO}$ has been deposited on a silica substrate. Its absorption $A_{0}$ is in the percent range and can be measured with the "VW" option of our Lambda 19 spetrophotometer from Perkin-Elmer: The spectrophotometer measures the reflection $R_{0}$ and the transmission $T_{0}$ of the coating. The multiple reflections coming from the silica/air interface and the silica/TaO layer have been taken into account in the calculation of $R_{0}$ and $T_{0} . A_{0}$ is deduced with the formula $A_{0}=1-R_{0}-T_{0}$. We measure $A_{0}=34200 \mathrm{ppm}$. A statistical study of the absorption of the standard has shown that $d A_{0}$ is $1000 \mathrm{ppm}$ so the uncertainty on $A_{0}$ is about $3 \% . \delta V$ is measured with a lock-in amplifier and $V$ with a voltmeter. An experimental study showed that the precision on these measurements is respectively $4 \%$ and $1 \%$. Thus, the uncertainty on the coating absorption measurement is about $13 \%$.

The purpose of our absorption bench is to be able to measure with a high accuracy the low loss coatings we are producing ( $0.1 \mathrm{ppm}$ range). Therefore, a sensitivity of a few tens of ppb is required. In our configuration, this sensibility can only be reached with a pump power higher than several Watts. Unfortunately, at this level of pump power, thermal lens effect occurs in the standard because of the high absorption. No more than a few hundreds milliWatts of pump power can be used. Consequently, a low absorption standard is necessary to reach the best sensitivity. For this reason, we coated a specific eleven quarter wavelength doublet $\mathrm{SiO}_{2}-\mathrm{Ta}_{2} \mathrm{O}_{5}$ mirror at $1064 \mathrm{~nm}$. We measured that the coating absorption of this low absorption standard is $5.0 \mathrm{ppm}$. With this standard, a pump power of $7 \mathrm{~W}$ can be used without any thermal lens effect. Of course, the uncertainty on the $5.0 \mathrm{ppm}$ is $13 \%$ as far as it is measured with the $34200 \mathrm{ppm}$ standard. With the same calculation as above, it can be estimated that the uncertainty measurement of a sample with the low absorption standard is $23 \%$. In fact, tens of measurements of the low absorption standard have been made in order to get an even better precision on the absorption value. With the choice of a low absorption standard, the uncertainty on the absorption measurement is decreased by less than a factor two, whereas the sensitivity is improved by more than a factor ten.
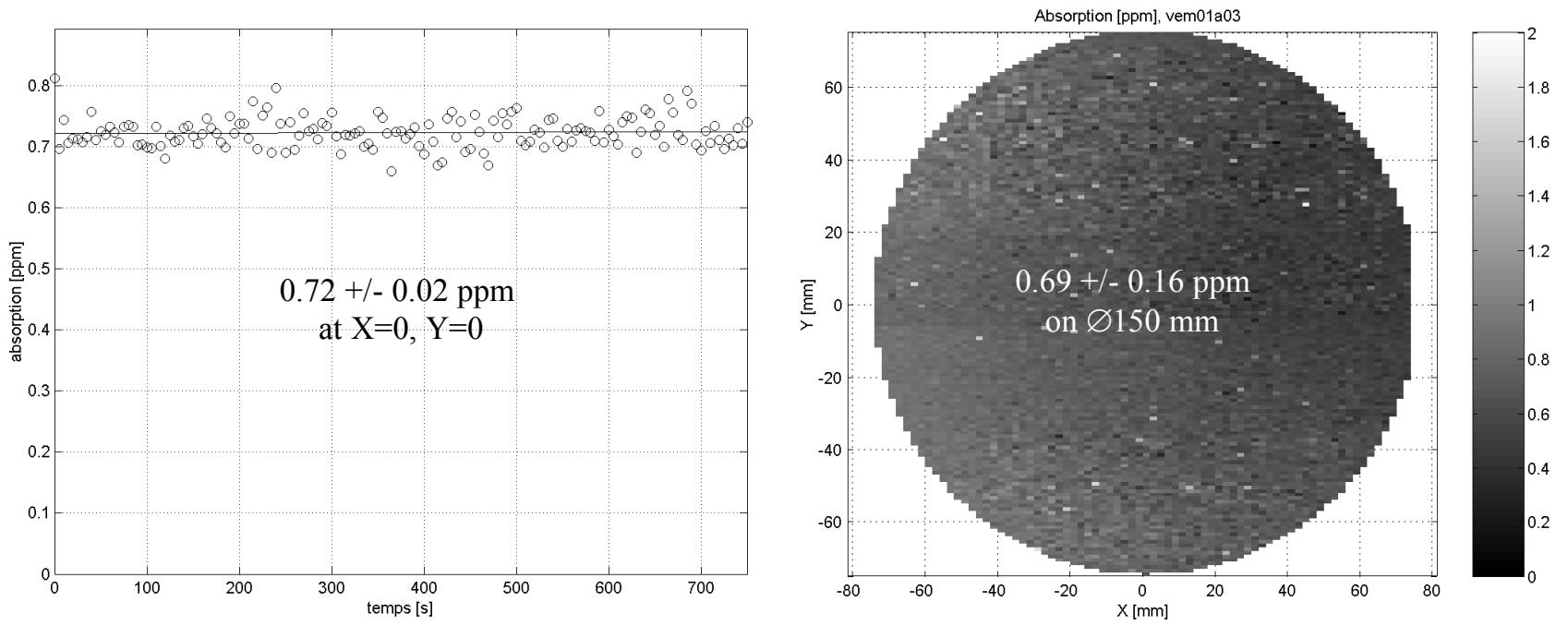


\subsubsection{Results}

Figure 3: Coating absorption vs time and absorption map of a VIRGO end mirror.

The absorption bench was first tested with the $\varnothing 350 \mathrm{~mm}$ end mirrors of the VIRGO interferometer we coated. At the center $(\mathrm{X}=0, \mathrm{Y}=0)$, we measured $0.72+/-0.02 \mathrm{ppm}$ during 12 minutes (Fig. 3). The standard deviation of the data distribution is $0.02 \mathrm{ppm}$. The noise threshold of the bench when the pump power is set to $7 \mathrm{~W}$ is also $0.02 \mathrm{ppm}$. The sensitivity of the coating absorption measurement is therefore $20 \mathrm{ppb}$. We also recorded a map of the absorption on 150 mm diameter. We measured $0.69+/-0.16 \mathrm{ppm}$. This result shows how stable the positioning system is and also how homogeneous the absorption of the mirror is.

\subsection{Bulk absorption}

\subsubsection{Standard}

At the present time, the bulk materials we are interested in are fused silica and sapphire. Silica and sapphire bulk absorption is far less than $1000 \mathrm{ppm} . \mathrm{cm}^{-1}$ at $1064 \mathrm{~nm}$ and can't be measured by a spectrophotometer as we did for the coating absorption standard. The characterization of a standard for each material requires another very well known material. We chose the water as far as it is has been very precisely and very often measured. A bibliographical study ${ }^{7}$ shows that pure water absorbs $13 \% . \mathrm{cm}^{-1}$ at $1064 \mathrm{~nm}$. At high frequency ( $3 \mathrm{kHz}$ in our case, which is the highest frequency of our mechanical chopper), the thermal diffusion length of the medium is much smaller than the pump waist size, and the deflection angle of the probe beam for a given pump power is proportional to ${ }^{5}$ :

$$
\left(\frac{d n}{d T}\right)_{m} \frac{\left[1-\exp \left(\alpha_{m} l\right)\right]}{\rho_{m} c_{m}}=K \cdot \frac{\delta V_{m}}{V_{m}}
$$

where

- $m$ is a medium such as water, silica or sapphire

- $\left(\frac{d n}{d T}\right)_{m}$ is the temperature coefficient of the index of refraction of the medium

$-\alpha_{m}$ is the optical absorption coefficient

$-l$ is the optical path length in the absorbing medium. In our case, it is the interaction length of pump and probe because the samples are longer than $3.4 \mathrm{~mm}$.

- $\rho_{m} c_{m}$ is the heat capacity per unit volume of the medium

- $K$ is a constant that does not depend on the medium

- $\delta V_{m}$ and $V_{m}$ are respectively the AC and DC voltages of the quadrant detector.

The constants used are summarized below (Tab.1).

\begin{tabular}{|c|c|c|c|}
\hline & Water & Silica & Sapphire \\
\hline$\frac{d n}{d T}\left[\mathrm{~K}^{-1}\right]$ & $9.10 \times 10^{-5}$ & $1.00 \times 10^{-5}$ & $13.3 \times 10^{-6}$ \\
\hline$\rho_{m}[\mathrm{~kg} \cdot \mathrm{m}-3]$ & 998.2 & 2202 & 3980 \\
\hline$c_{m}\left[\mathrm{~J} \cdot \mathrm{kg}^{-1} \cdot \mathrm{K}^{-1}\right]$ & 4182 & 771 & 760 \\
\hline
\end{tabular}

Table 1: physical constants used for water, silica and sapphire. 
Then, it is possible to characterize a silica standard as well as a sapphire standard, from the ultra pure water:

$$
\alpha_{\text {silica }}\left[\text { ppm.cm }{ }^{-1}\right]=4.5 \times 10^{5} \cdot \frac{\delta V_{\text {silica }}}{V_{\text {silica }}} \cdot \frac{V_{\text {water }}}{\delta V_{\text {water }}} \quad \alpha_{\text {sapphire }}\left[\text { ppm.cm }{ }^{-1}\right]=0.60 \times 10^{5} \cdot \frac{\delta V_{\text {sapphire }}}{V_{\text {sapphire }}} \cdot \frac{V_{\text {water }}}{\delta V_{\text {water }}}
$$

Experimental conditions for the measurement of $\delta V$ and $V$ with the water and with the standard have to be exactly the same. Therefore, it is not possible to use high pump power because the water is absorbing very much: the water boils if the pump power exceeds $40 \mathrm{~mW}$. We used ultra pure water that we are producing (reverse osmosis technique and demineralization). The water is put in a quartz tank. Because of the low pump power required, it is preferable not to use a to low absorbing standard. For example, the silica standard we characterized is absorbing $90 \mathrm{ppm} . \mathrm{cm}^{-1}$. The uncertainty on this value is about $10 \%$ and can be estimated with the same method as for the coating absorption. Thus, the uncertainty on a bulk absorption measurement of a silica or a sapphire sample using the previous standards is about $20 \%$.

\subsubsection{Results}

The bulk absorption bench has been tested with $\varnothing 350 \mathrm{~mm}$ silica VIRGO optics and also with a $\varnothing 314 \mathrm{~mm}$ experimental LIGO sapphire optic. With $30 \mathrm{~W}$ pump power, a sensitivity of $20 \mathrm{ppb} . \mathrm{cm}^{-1}$ is achieved. Figure 4 shows the bulk absorption of a VIRGO input mirror out of Suprasil 312 SV from Heraeus. It has been measured during 10 minutes at the center of the sample. It is absorbing no more than $0.62 \mathrm{ppm} . \mathrm{cm}^{-1}$ and the standard deviation of the data distribution is $0.02 \mathrm{ppm} . \mathrm{cm}^{-1}$ (Fig. 4). A map on $\varnothing 150 \mathrm{~mm}$ shows the uniformity of the bulk absorption : $0.57+/-0.12 \mathrm{ppm} . \mathrm{cm}^{-1}$.
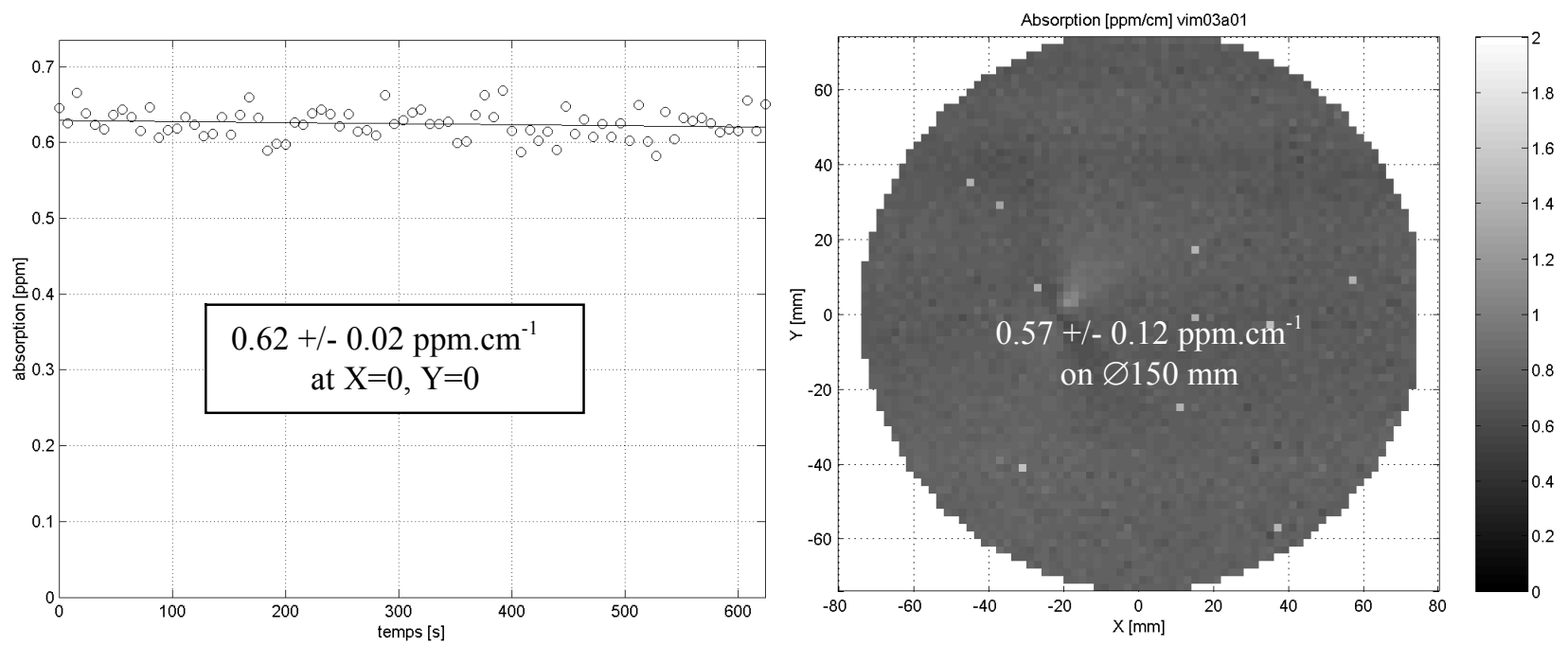

Figure 4: Bulk absorption vs time and absorption map of a silica VIRGO optic.

More interesting, from a metrological point of view is the sapphire. On a $\varnothing 200 \mathrm{~mm}$ map, it can be noticed that the absorption varies from 40 to $140 \mathrm{ppm} . \mathrm{cm}^{-1}$ (Fig. 5 left). If a zoom is made on a pixel a this map, absorption variations in the $100 \mu \mathrm{m}$ scale are observed (Fig. 5 right).
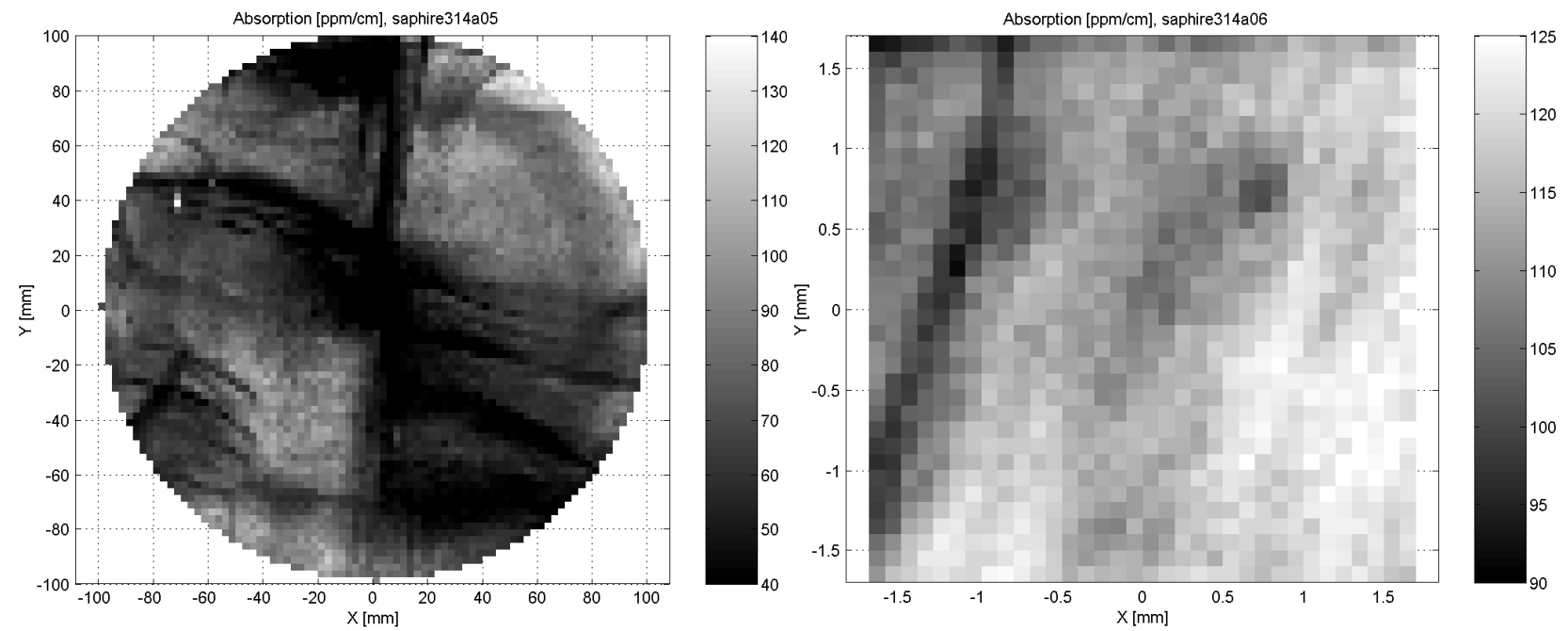
Figure 5: Bulk absorption maps of an experimental LIGO sapphire optic.

Another feature of the absorption bench is that it enables bulk absorption measurement versus the depth of the sample. Here we can see absorption variations from 67 to $127 \mathrm{ppm} . \mathrm{cm}^{-1}$ along the $132 \mathrm{~mm}$ depth of the sapphire optic (Fig. 6).

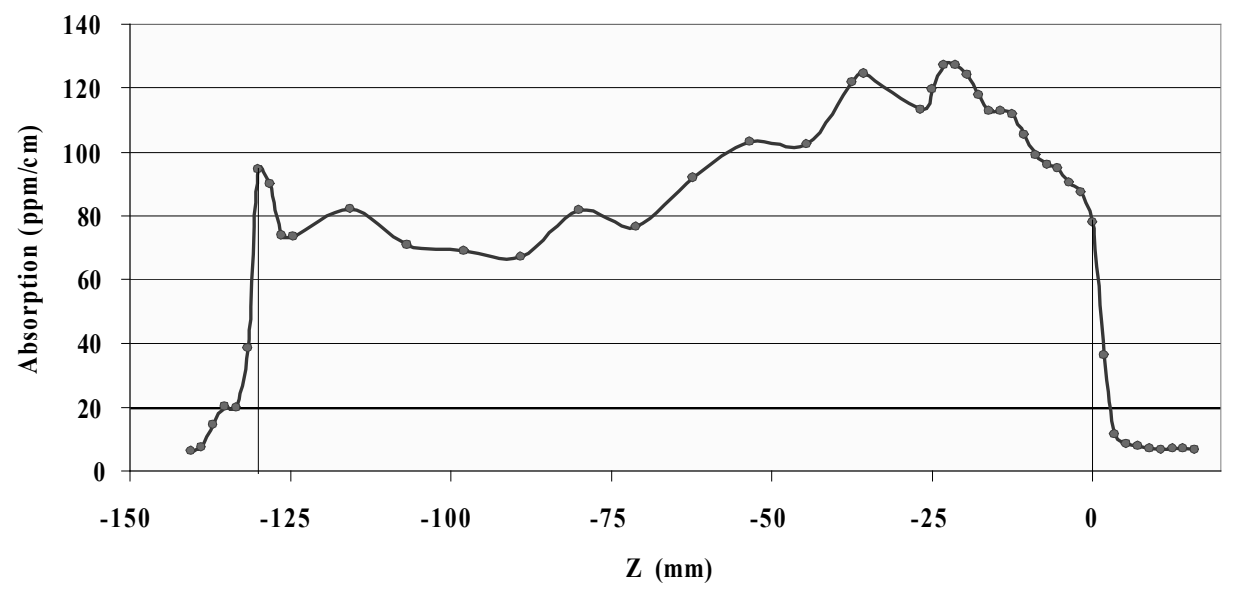

Figure 6: Bulk absorption of an experimental LIGO sapphire optic vs depth.

\section{BIREFRINGENCE MEASUREMENT}

\subsection{Experimental setup}

The principle of this birefringence bench has been developped at ESPCI Paris ${ }^{8}$ and has been implemented and futher developped in our laboratory. The schematic of the bench is presented on figure 7. The light source is a $830 \mathrm{~nm}$ LED and the working frequency of the modulator is $\mathrm{f}=50 \mathrm{kHz}$. Two lock-in detectors are connected to photo-detector in order to detect the modulated signal at $\mathrm{f}$ and $2 \mathrm{f}$. This allows the measurement of both the birefringence amplitude and the direction of the principle axis. The sample is motioned by the same positioning system as the one used for the absorption bench.

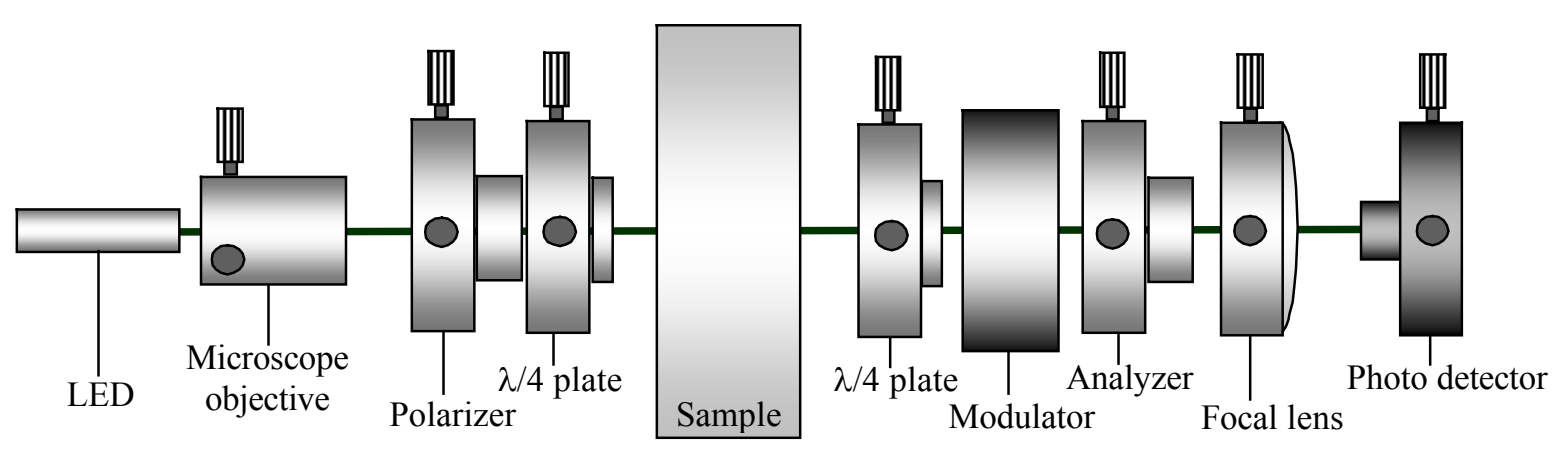

Figure 7: Experimental setup of the birefringence bench. 


\subsection{Results}

The sensitivity of the bench is about $5.10^{-5} \mathrm{rad}$ with the translation stages motion on and with $2 \mathrm{~s}$ data integration. Here again, the bench was tested with the VIRGO large silica optics and with the experimental LIGO sapphire optic. In the VIRGO silica sample, the birefringence varies from 0.02 to 5.7 mrad.cm ${ }^{-1}$ (Fig. 8 left). In fact, the birefringence is very sensitive to the pressure of the mount on the silica. This is the reason why the birefringence is very high at the edges of the sample where the pressure is the highest. In the center region of the sample, 0.02 mrad may be considered as the maximum intrinsic birefringence amplitude. In the VIRGO experiment, the birefringence mainly comes from the holding system of the mirror. With our bench, it is possible to simulate the holding system of VIRGO and estimate the real birefringence occurring during the experiment. The case of the experimental LIGO sapphire is different because the sapphire is a crystal. The birefringence was measured along the optical c-axis. The variation of the birefringence is very small: from 0.57 to 0.84 mrad. $\mathrm{cm}^{-1}$ (Fig. 8 right). It first means that the crystal is regular. Then, we observe that sapphire is harder than the silica and thus much less sensitive to the pressure of the mount. Finally, the map shows that the positioning system is very good: if the rolling of the stages was too high, the beam would not stay precisely along the c-axis and the birefringence would vary very much during the mapping.
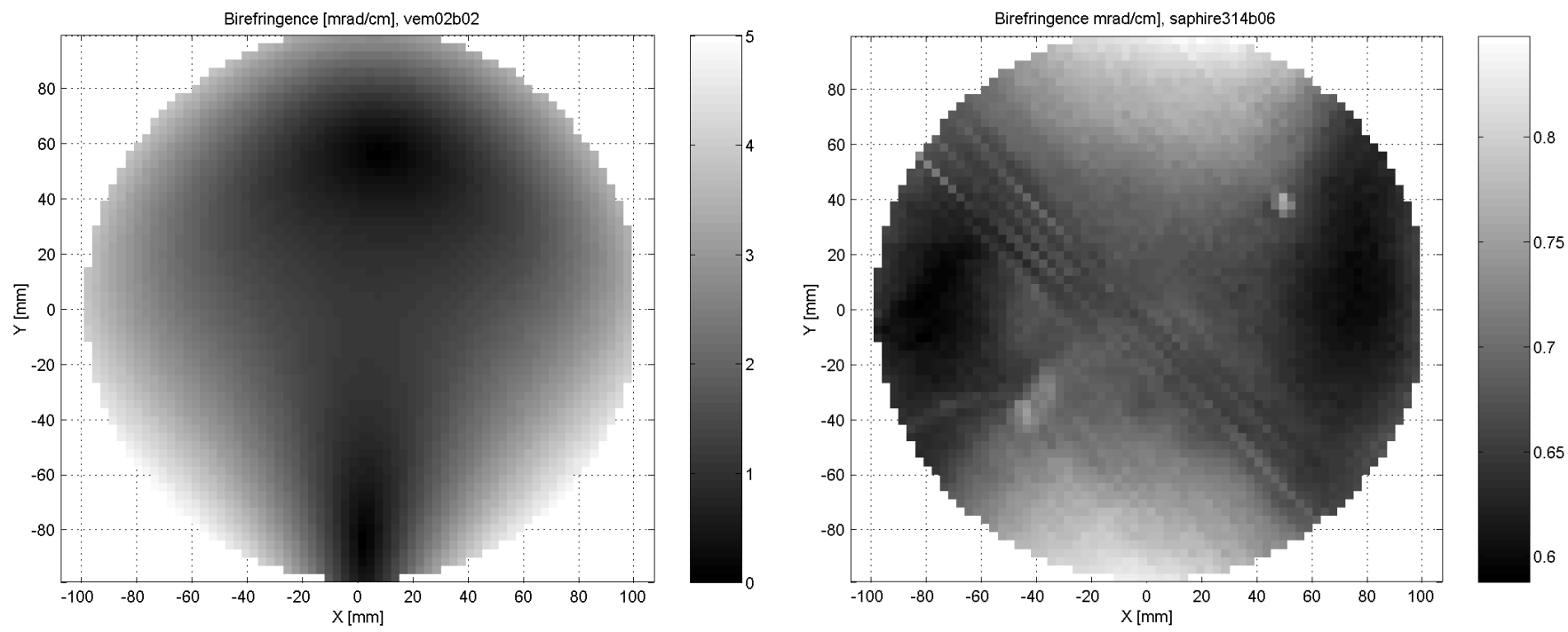

Figure 8: Birefringence map of a VIRGO silica optic (left) and of an experimental LIGO sapphire optic (right).

\section{LARGE WAVEFRONT MEASUREMENT}

We use a commercial interferometer from ADE Phase Shift (minifiz type interferometer, pupil $\varnothing 150$ mm, wavelength $1064 \mathrm{~nm}$ ). This interferometer has a very good stability as a function of time and a remarkable reproducibility (difference between two successive measurements): $\mathbf{0 . 0 4} \mathbf{~ n m ~ R M S ~ ( F i g u r e ~ 9 ) . ~ T o ~ m e a s u r e ~ l a r g e ~ w a v e f r o n t , ~ w e ~ h a v e ~}$ developed, in collaboration with a French company (MB Optique), a numerical method called "Stitching Interferometry" 9,10 .

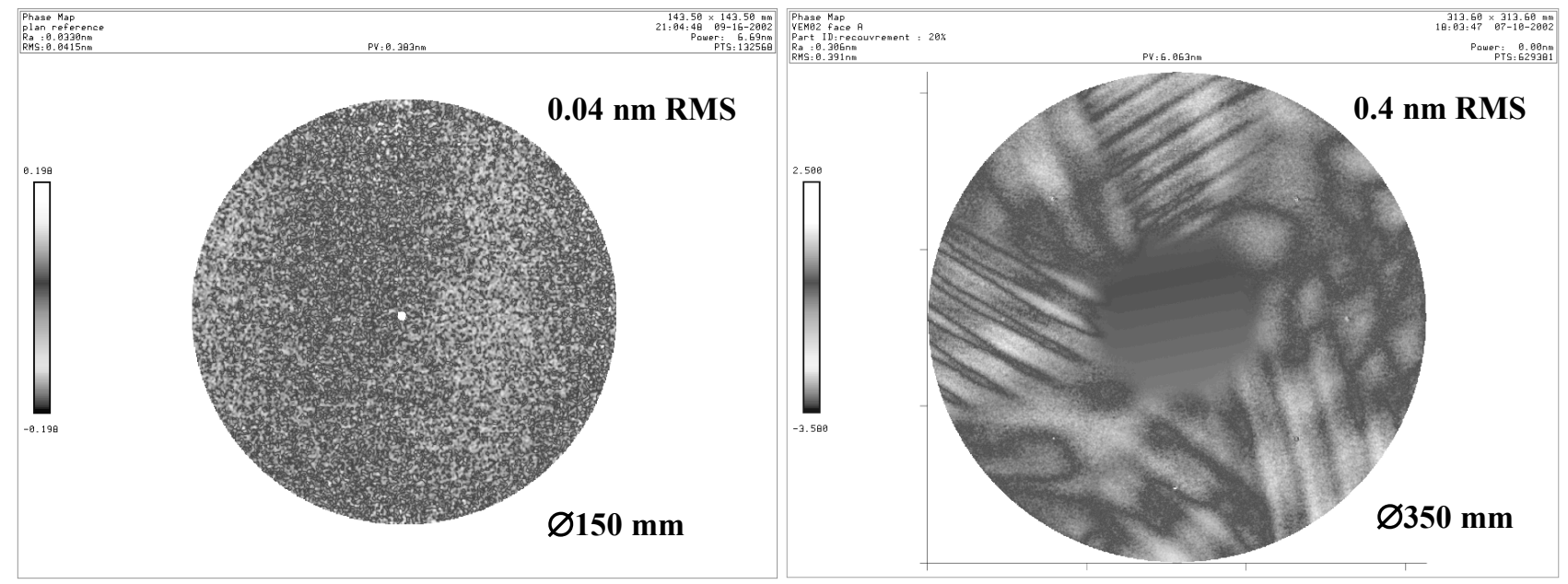


Figure 9 : Reproducibility of the interferometer with (right) and without (left) stitching option

Stitching interferometry enables the measurement of large optical components using a standard "small" interferometer. This result is obtained by taking multiple overlapping images of the large component, and numerically "stitching" these sub-apertures together by computing a "Tip-Tilt-Piston" correction for each sub-aperture. A large sample holder (gimbal) is necessary to move the optical component in front of the interferometer. Severe requirements were defined concerning the accuracy $(\# 1 \mu \mathrm{m})$ and the reproducibility of the displacement. We use the same positioning system as the one for absorption measurements. Thus, wavefront measurements up to $\varnothing 400 \mathrm{~mm}$ are achievable. The reproducibility of this method is $\mathbf{0 . 4} \mathbf{~ n m ~ R M S ~ o n ~} \mathbf{8 4 0 0} \mathbf{~ m m}$ : this is remarkable regarding the measurement complexity (Figure 9). When we subtract two successive measurements, residual fringes are visible. The principal error source comes from the residual vibrations, which are not entirely filtered at the moment on the optical table. We are obliged to put the interferometer $60 \mathrm{~cm}$ above the optical table to measure large samples, which is not a favorable case. These vibrations are generated during the displacement of the mirror gimbal from one zone to the other. A better damping should be found in order to improve even more the method sensitivity. Improvements should occur in the next future, but we can already measure wavefronts of large samples (Figure 10).

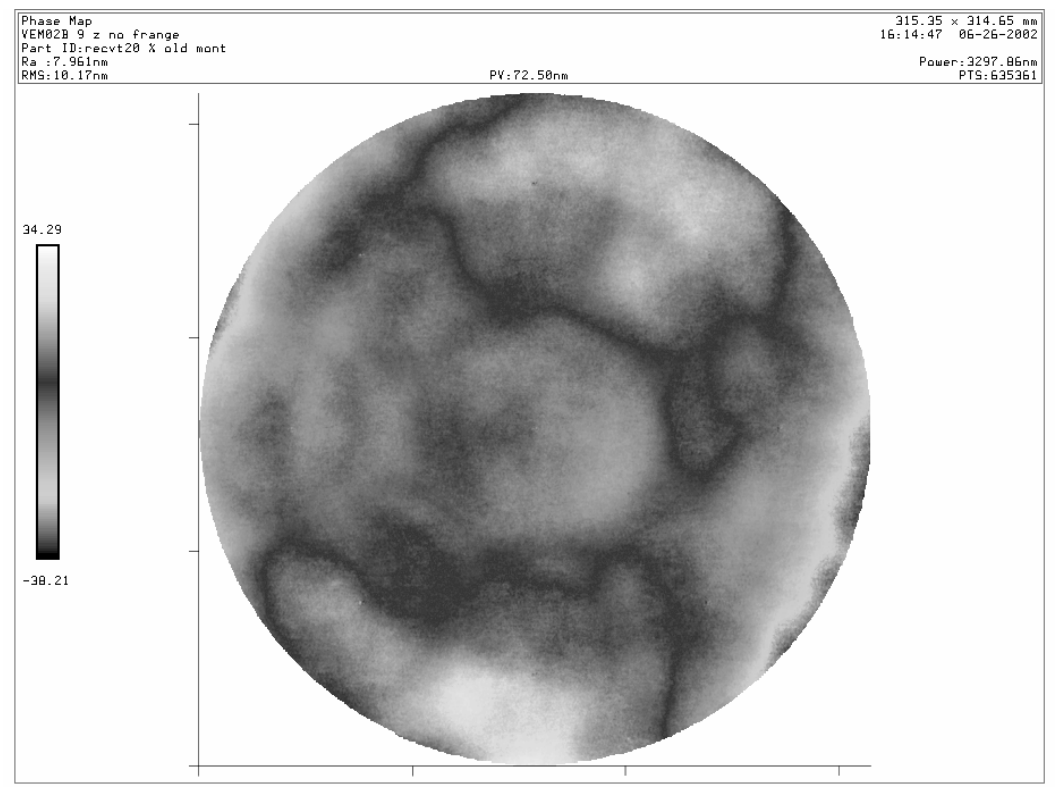

Figure 10: Wavefront on $\varnothing 315 \mathrm{~mm}$ of a large IBS mirror (stitching with 9 zones)

\section{ROUGHNESS MEASUREMENT AND DEFECTS DETECTION}

We use a commercial profilometer MICROMAP, which was modified to characterize large optics. Different interferometric objectives (5X,10X, 20X, 50X) are used. That allows measurement area between $1 \mathrm{~mm}^{2}$ and $50 * 50 \mu \mathrm{m}^{2}$ and the measurement sensitivity is $0.2 \AA$ RMS. The profilometer is limited to the measurement of small spatial periods $(<<1 \mathrm{~mm})$, contrary to the Phase Shift interferometer, which is sensitive to spatial periods greater than several 
millimeters (pixel size $350 \mu \mathrm{m}$ ). Thus, to be able to characterize spatial periods of a surface between $500 \mu \mathrm{m}$ and $5 \mathrm{~mm}$, we have developed a stitching option on our profilometer to evaluate the roughness on large area. Two $400 \mathrm{~mm}$ linear table were installed below the Micromap to move the sample (X, Y movement). Figure 11 shows two examples of such measurements on large area: $4.1 * 4.1 \mathrm{~mm}^{2}$ (81 zones coupled) and $2.9 * 2.9 \mathrm{~mm}^{2}$ (49 zones coupled). The micropolished substrate has a remarkable RMS roughness (0.38 $\AA$ RMS). Nevertheless, very thin scratches are visible on this large area thanks to the stitching method. On the rough substrate (18.6 nm RMS), a periodic shape was pointed out. These two results show how useful the stitching measurement on the profilometer Micromap is. We are able now to see for instance periodic defects, long thin scratches.

The scattering level is not depending only on the Rayleigh diffraction theory linked to the surface roughness $\sigma$ by the formula: TIS $=(4 \pi \sigma / \lambda)^{2}$, where TIS means Total Integrated Scatter and $\lambda$ is the wavelength. It depends also on the number and the size of the point defects on the surface. So, we have developed, in collaboration with Micromap, a unique prototype around the profilometer capable to detect small defects (detection threshold $\varnothing 0,3 \mu \mathrm{m}$ ). For this measurement, we use a standard dark field objective. We can qualify the substrate surface quality (maps on $\varnothing 400 \mathrm{~mm}$ ) and also the substrate cleaning procedure, which is a crucial part of our deposition process.

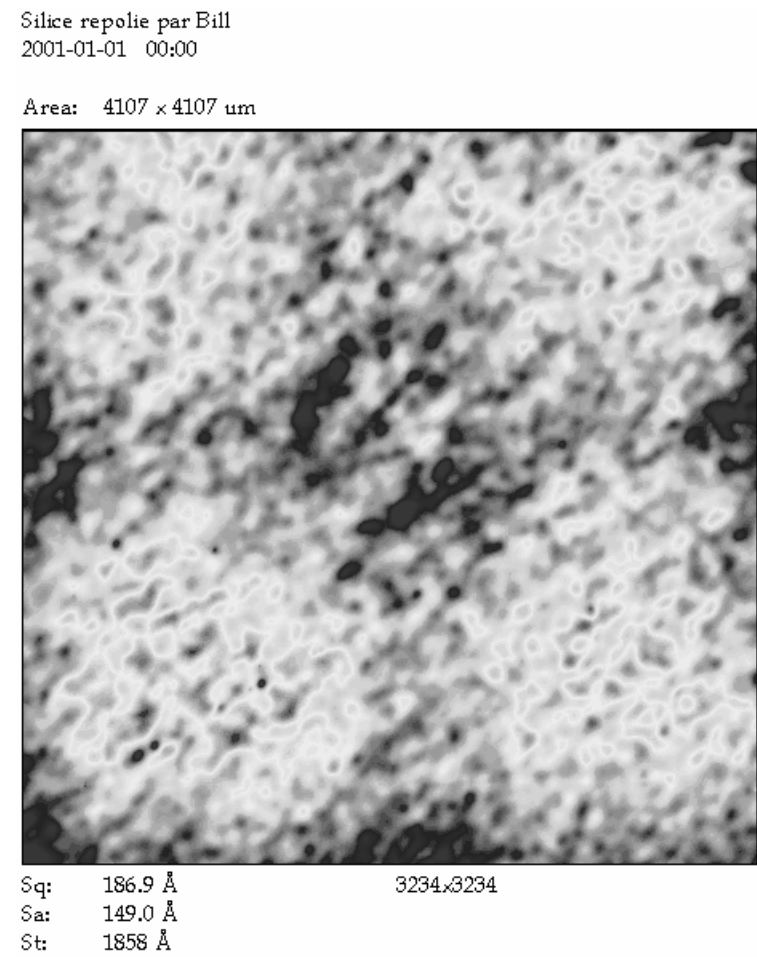

Silice micropolie - Sub recyclage CITF 2001-01-01 00:00

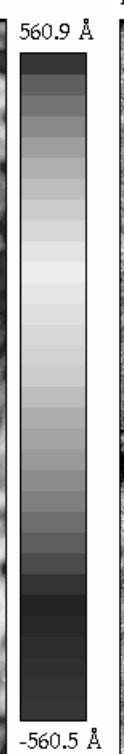

Area: $2907 \times 2907 \mathrm{um}$

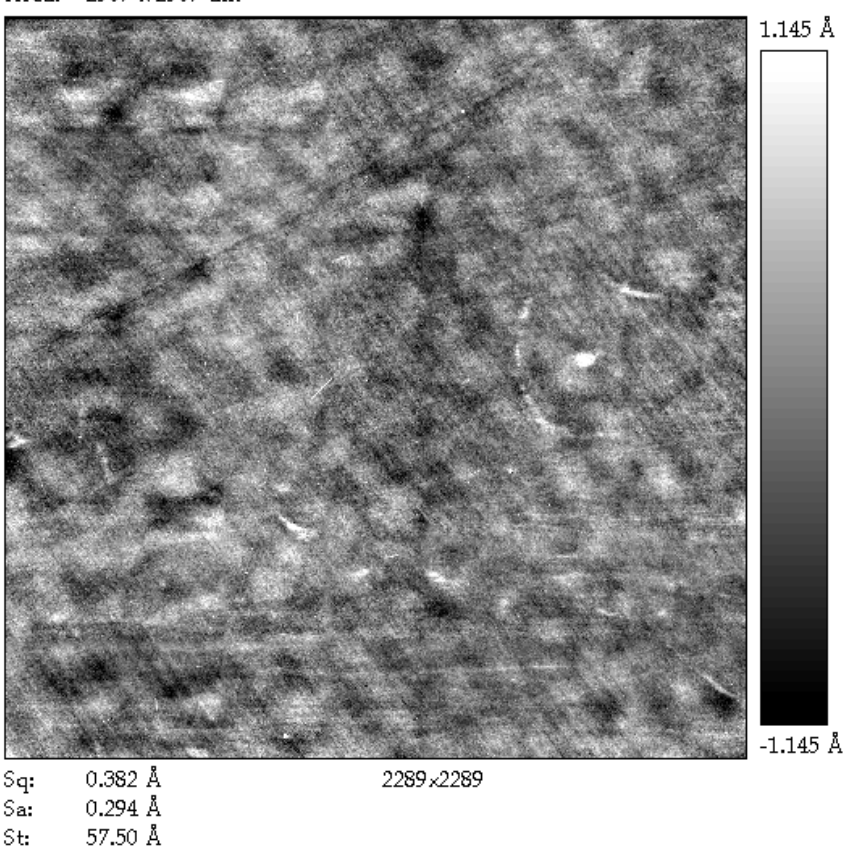

Figure 11: Roughness measurement on large area of a silica substrate having an 'orange' skin (left) and of a micropolished silica substrate (right)
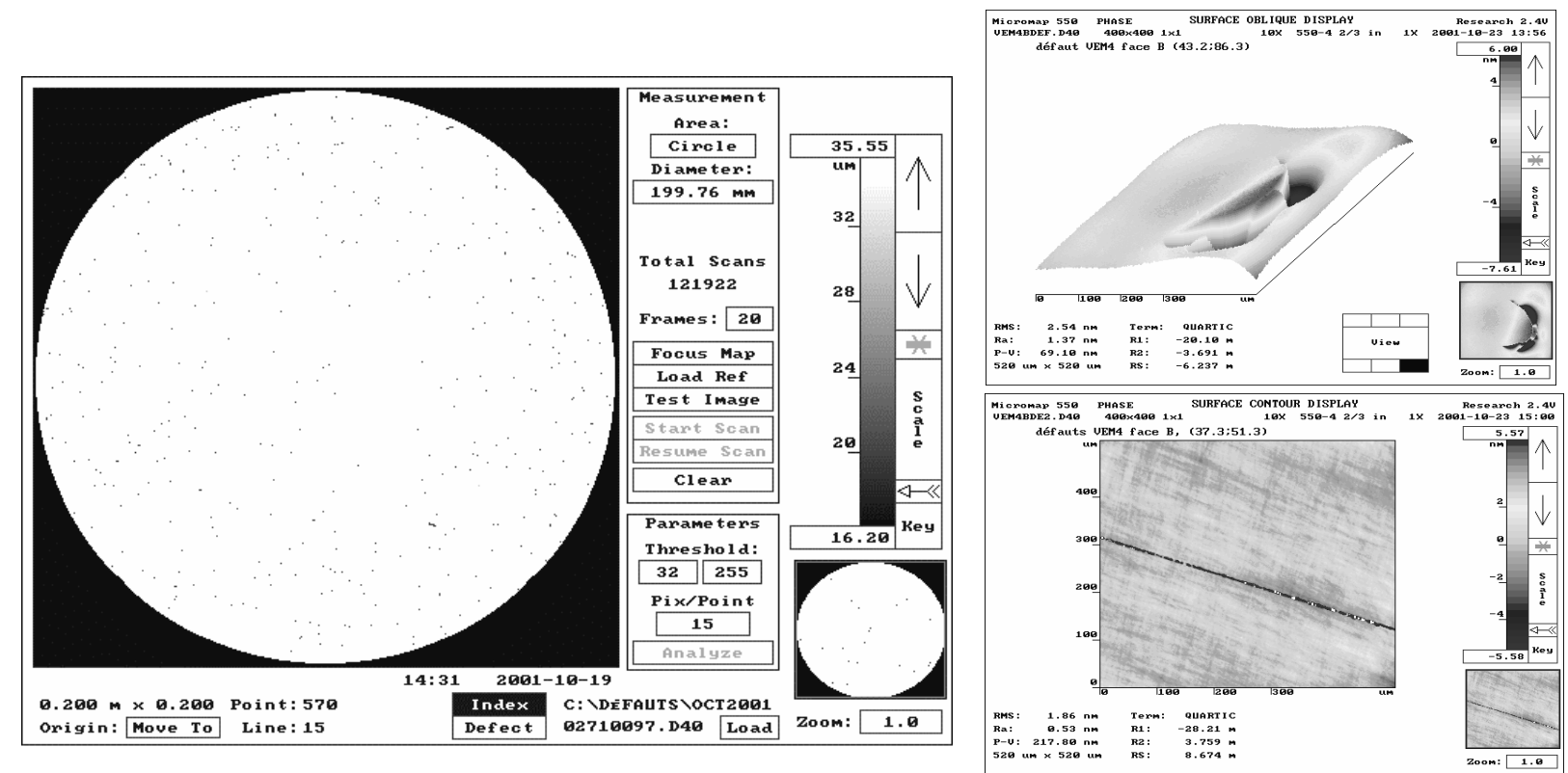
Figure 12: Defects detection measurement on $\varnothing 200$ and two examples of detected defects.

This method distinguishes small defects $(0.3-1 \mu \mathrm{m})$, larger defects $(>1 \mu \mathrm{m})$ and scratches. Figure 12 shows an example of such a detection on a $\varnothing 350 \mathrm{~mm}$ substrate. The measurement lasts a very long time (more than 100000 images $400 * 400 \mu \mathrm{m}^{2}$ measured to map a diameter of $200 \mathrm{~mm}$ ). This implies that the environment around the system is very clean to avoid contamination during the measurement. That is why the Micromap is installed in the Class 1 clean room.

\section{CONCLUSION}

A new metrology facility for low loss large optics has been developed at SMA-VIRGO. Five original benches, placed in a class 1 clean room, allow the characterization of the optical losses of components up to $\varnothing 400 \mathrm{~mm}$ coated by Ion Beam Sputtering. The two main characteristics of these benches are their sensitivity and their size; the mapping of the whole $\varnothing 400 \mathrm{~mm}$ surface of the component is possible on every measurement bench:

A scatterometer is able to measure scattering maps with a sensitivity better than $1 \mathrm{ppm}$. It can distinguish the scattering coming from the surface from the scattering of the bulk material. This system can also measure transmission maps with a sensitivity also better than $1 \mathrm{ppm}$.

An absorption bench allows the measurement of coating absorption mappings with a sensitivity of $20 \mathrm{ppb}$ and an uncertainty on the measurement of about $23 \%$.This is achieved thanks to a low loss absorption standard of 5.0 ppm. The bench also enables bulk absorption with a sensitivity of $20 \mathrm{ppb} . \mathrm{cm}^{-1}$ and an uncertainty on the measurement of about $20 \%$. In this case, the calibration of the standard has required a study of the absorption of ultra pure water.

A birefringence bench can measure the amplitude of the birefringence with a sensitivity of $5.10^{-5}$ radian and the direction of the principle axis. During a birefringence mapping, we checked with a sapphire crystal that the quality of the measurement is not limited by the positioning system of the sample which is very accurate.

A control interferometer equipped with an original stitching option is able to measure wavefronts on $400 \mathrm{~mm}$ diameter with a repeatability of $0.4 \mathrm{~nm}$ RMS. The sensitivity of the measurement is limited by vibrations generated during the displacement of the mirror positioning system from one zone to another. We are working on an improvement of the damping system in order to reach an even better sensitivity.

Finally, a profilometer allows the measurement of the roughness with a sensitivity of $0.2 \AA$ RMS. The measured area depends on the different interferometric objectives used (from $50 \times 50 \mu \mathrm{m}^{2}$ to $1 \mathrm{x} 1 \mathrm{~mm}^{2}$ ) and can be extended to more than $5 \times 5 \mathrm{~mm}^{2}$ with a stitching technique, the limiting factor being the time required for the measurement. A prototype using this profilometer has been developed in order to detect small defects on surfaces. This technique enables the detection of defects as small as $0.3 \mu \mathrm{m}$ on sample as large as $400 \mathrm{x} 400 \mathrm{~mm}^{2}$.

\section{REFERENCES}

1. L. Pinard, P. Ganau, J.M. Mackowski, C. Michel, M. Napolitano, E. Vireton, A.C. Boccara, V. Loriette, H. Piombini, “95'Optical Interference Coatings”, Technical Digest Vol. 17, p 200-203, Tucson, 1995.

2. J.M. Mackowski, L. Pinard, L. Dognin, P. Ganau, B. Lagrange, C. Michel, M. Morgue; “98'Optical Interference Coatings", Technical Digest Vol. 9, p 18-20, Tucson, 1998.

3. J.C. Stower, McGraw-Hill, NY 1990.

4. J.C. Stower, D.E. McGary, Proceeding SPIE, p 967,1988.

5. A.C. Boccara, D. Fournier, W. Jackson and N.M. Amer, "sensitive photothermal deflection technique for measuring absorption in optically thin media", Optics Letter, 5, 377-379, 1980.

6. W.B. Jackson, N.M. AMER, A.C. Boccara and D. Fournier, "Photothermal deflection spectroscopy and detection", Applied Optics, 20 No 8, 1333-1344, 1981.

7. http://omlc.ogi.edu/spectra/water/abs/ 
8. Geraud de Villele and Vincent Loriette, "Birefringence imaging with imperfect benches: application to large scale birefringence measurements" Applied Optics, 39, 3864-3874, 2000.

9. $\quad$ M. Bray, SPIE Vol.3739, p 259-273, 1999.

10. M. Bray, SPIE Vol 4451, H. Philip Stahl (Ed.), 2001. 